\title{
Philonsorbonne
}

12 | 2018

Année 2017-2018

\section{Présentation : rationalité pratique et motivation morale dans l'éthique des vertus et l'éthique " existentielle »}

\section{Olivier D'JERANIAN et Yoann MALINGE}

\section{CpenEdition}

\section{Journals}

Édition électronique

URL : https://journals.openedition.org/philonsorbonne/992

DOI : 10.4000/philonsorbonne.992

ISSN : 2270-7336

Éditeur

Publications de la Sorbonne

\section{Édition imprimée}

Date de publication : 1 janvier 2018

Pagination : 105-106

ISSN : 1255-183X

Référence électronique

Olivier D'JERANIAN et Yoann MALINGE, «Présentation : rationalité pratique et motivation morale dans l'éthique des vertus et l'éthique « existentielle » », Philonsorbonne [En ligne], 12 | 2018, mis en ligne le 18 mars 2018, consulté le 10 juin 2021. URL : http://journals.openedition.org/philonsorbonne/992 ; DOI : https://doi.org/10.4000/philonsorbonne.992 


\title{
Présentation : \\ Rationalité pratique et motivation morale dans l'éthique des vertus et l'éthique « existentielle »
}

\author{
Olivier D'JeRANIAN et Yoann MaLINGE
}

Dans le précédent numéro de Philonsorbonne, une première partie du dossier «Rationalité pratique et motivation morale» avait été publiée. Il s'agissait de rendre compte des travaux menés dans le cadre du projet unissant deux centres de recherches: le Centre de Philosophie Contemporaine de l'Université Paris 1 Panthéon-Sorbonne et l'Instituto de Filosofia Pratica de l'Universidade da Beira Interior au Portugal.

Le projet intitulé «Rationalité pratique, jugement et motivation morale » a pu recevoir le soutien du programme PHC Pessoa (308448UF). Il consistait à étudier les formes du jugement pratique et le rôle des raisons morales au travers de l'éthique des vertus d'une part, et d'une éthique «existentielle» d'autre part. La première partie du dossier contenait trois articles qui s'intéressaient à l'éthique des vertus telle qu'elle se trouve notamment développée par Aristote et les Stoïciens.

Le présent numéro de Philonsorbonne accueille la seconde partie de ce dossier. Il regroupe trois articles consacrés à l'éthique des vertus pour l'un et à l'éthique « existentielle » pour les deux autres.

- Dans un premier article, Rui Sampaio da Silva interroge la motivation morale dans l'éthique de la vertu. En tenant compte de la pluralité des motivations, il faut reconnaître la difficulté d'établir, avec Aristote, la relation entre la raison et les désirs dans la motivation morale. L'éthique aristotélicienne a connu diverses interprétations sur la nature de la vertu éthique. L'objectif de cet article n'est pas d'entreprendre une exégèse de la théorie d'Aristote mais de mener une recherche qui, loin de séparer les facteurs cognitifs et volitifs, cherche plutôt à les combiner. Les désirs ne sont pas une force causale brute, ils possèdent un caractère rationnel. Dès lors, il faut analyser le rôle de l'éducation morale comme processus d'acquisition d'un cadre d'interprétation qui façonne la perception de la réalité. 
À partir de cette compréhension, l'article s'intéresse à la nature du jugement moral. Dans l'éthique de la vertu, s'il y a bien des principes moraux, ceux-ci admettent des exceptions. C'est alors qu'il faut saisir la teneur de la sagesse pratique, son origine dans l'expérience et la fiabilité de son rôle dans le jugement moral.

- Dans un deuxième article, André Barata interroge la philosophie sartrienne en tant que théorie de l'action. À partir d'une compréhension de la liberté comme n'étant pas celle du libre-arbitre, il s'agit de montrer que la volonté n'est pas le moteur de l'action humaine et que c'est plutôt à la vie émotionnelle qu'il faut accorder un rôle central. Les objets du monde se présentent avec des significations différentes selon les émotions vécues. Interrogeant la différence entre motifs et mobiles, l'auteur montre qu'ils sont corrélatifs du projet.

Dès lors, André Barata développe l'hypothèse qu'il y a une éthique déontologique chez Sartre différente de celle de Kant, notamment en ce que l'universalité n'est pas une condition contraignant le choix mais le résultat co-extensif de la radicalité d'un choix singulier.

Enfin, à partir d'un examen des traits de caractère qui sont corrélatifs du projet existentiel, il est possible à l'auteur de mener une comparaison avec l'éthique de la vertu, même si la perspective de Sartre est opposée à celle d'Aristote.

- Dans un troisième article, Yoann Malinge interroge le rapport de l'agent à sa motivation dans la philosophie de Sartre. À partir de l'analyse de l'angoisse comme expérience de la liberté, qu'elle soit angoisse devant l'avenir ou devant le passé, il s'agit d'examiner comment sont vécues les différentes possibilités d'action. La compréhension ontologique de l'humain comme pour-soi et de la conscience comme intentionnalité conduit l'agent à envisager ses possibilités d'agir comme séparées de lui par un néant.

L'habitude semblerait alors constituer un contre-exemple puisqu'elle propose une possibilité d'action facilitée par sa répétition antérieure. Opposant Sartre à Ravaisson, cet article montre que l'habitude n'est pas pensée comme une possibilité privilégiée de l'action d'un homme, selon l'auteur de L'être et le néant. Yoann Malinge cherche alors à réinscrire le phénomène de l'habitude dans la perspective du projet originel. Ainsi s'agit-il de montrer que la liberté est vécue dans un engagement existentiel, compris par contraste avec la liberté d'indifférence. L'agent peut accorder une valeur différente aux motifs qui l'entraînent à choisir une action plutôt qu'une autre, selon son projet originel. Chaque être humain est responsable de son projet, mais sa liberté pour le modifier n'est pas absolue. 